\title{
Messages to first responders from a bereaved parent
}

\author{
Richard Brown, THINK Hauora Primary Health Organisation
}

\begin{abstract}
INTRODUCTION: This article describes the use of autoethnography to explore my experience as a bereaved parent in relation to the traumatic loss of my 12-year-old child in 2009.

METHODS: Employing an autoethnographic approach, this research articulates and investigates personal narratives associated with experiences of the death of my young child. Narratives offer a rich description of the event and allow data analysis where emerging themes stream from a personal experience.
\end{abstract}

FINDINGS: Professionals taking control and the caring role of the community are main themes identified and analysed from these personal narratives. The findings advocate for an understanding of the needs of grieving parents at a time of extreme confusion. This article concentrates on professional first responders taking control and guiding traumatised parents when their child has a fatal event. Bereaved parents experience disorientation and traumatic confusion at the sudden death of their child. Emergency responders need to offer active listening skills and guidance.

IMPLICATIONS: Professional first responders need to take control of the bereaved parent's early journey of grief as they are likely to be in a state of deep traumatic shock, where disorientation leaves them vulnerable to lowered cognitive ability. The use of active listening skills and sensitivity can direct traumatised parents, thereby assisting the short- and long-term welfare of the family by addressing their initial needs at a time of crisis.

KEYWORDS: Autoethnography; bereaved parents; first responders; professionals taking control; emergency services; bereavement support

One night in March 2009, I was thrown into a world of personal trauma and grief through the sudden death of my young child. A search for meaning to the event led me to research and analyse a dual insider experience. I was walking the experience of a bereaved and traumatised parent, as a registered social worker in Aotearoa New Zealand who had previously responded to other people's traumatic events.

Amongst many social work theories, one is the theory of crisis intervention. Regehr
(2011) describes crisis as a period where an individual is unable to cope as usual due to a psychological imbalance; an individual can have impaired ability to perform tasks or to communicate. When working in the emergency department of a hospital, I have provided support for families affected by trauma and grief at the sudden loss of a family member. This task requires patience and empathy for a social worker to appreciate that individuals may be experiencing shock and confusion. It is most important that a social worker does 
not assume they understand the person's experience.

Janzen et al. (2004) explained how emergency professionals cannot underestimate the effect of traumatic bereavement on a parent. As a professional social worker, I had little knowledge of how the death of my child would affect me. I did not know what to expect, either about my own emotional reactions or other people's reactions. I expected that the professional encounter would be kind, compassionate, and helpful. I assumed that professionals know what to do in a crisis, are trained to handle traumatic events. Yet I encountered some emergency first responders who were proficient and some who were not. I felt vulnerable and the reactions of others had a significant impact on me. I can remember words, said and done which were comforting and, even more, actions, said and done that were hurtful and upsetting. Janzen et al. (2004) supported this parental perspective in their research.

This article focuses on the main research question: "As a bereaved parent, what messages are important for emergency response personnel when responding to a request for help to a dying child and their parents who live in a rural community in Aotearoa New Zealand?" The purpose of the study is to create awareness of the experience of a traumatised and bereaved parent for emergency first responders called to an event where a child has died. Dyregrov and Dyregrov (2008) and Feigelman et al. (2012) referred to the phenomenon of losing a child as a devastating experience for a parent. Such traumatic impact can result in complicated grief due to the parent-child attachment and this may include witnessing the event. So, what can emergency responders learn when attending the scene of such a devastating event?

Emergency personnel responding to a traumatic incident involving a child are responsible for managing, not only the child, but the response of the parents. Aho et al.
(2010) explained the follow-up intervention for grieving parents, being skills of affect, aid and affirmation. Parents need guidance through post-incident tasks and to feel that people are listening throughout such processes.

Emergency responders need to ensure that parents understand how and why processes may occur and what role they have, and to challenge them if necessary. Fulbrook (2015) noted that a parent must start processing the psychological reality of the death of their child. Parents have shattered assumptions of believing their child would outlive them.

The lens of my personal experience offers a rich narrative description of an event. Narrative is knowledge that captures the knowing and telling of such an event (Kim, 2016). This research facilitates an inside perspective that would otherwise not be captured (Pace, 2012). I thus reflect on a very personal experience, that enables an understanding of events that may occur for parents when a child dies and contributes to the research question: "What should first responders offer to traumatised parents?"

I have recently observed the 12th anniversary of the death of my child. The passage of time has allowed a reflective personal journey of grief. This research started as a quest for meaning into the loss from the beginning moment of questioning, "why me?" Severe shock engulfed close family members who could not comprehend this loss. The study has been a continuous iterative learning process along a learning path of grief; arriving at a place where I have been able to share my story, adding to the research of parental grief.

\section{Methodology}

This is a study of the experience of the death of my child. In writing about this phenomenon, I seek to interpret a meaning to my loss, approaching the research from constructivism, where my knowledge develops through experiences and 
interactions. O'Hara et al. (2014) asserted that social phenomena, and their interpretation, are revised through people's interaction with each other.

The research method for this study crossed methodological boundaries of ethnography, phenomenology, and narrative inquiry in the complexity of writing a personal and evocative autoethnographic study. Autoethnography is the research method chosen for this self-investigatory task due to its ability to help an inside perspective that would otherwise remain unfamiliar (Pace, 2012). Mendez (2013) describes autoethnographic method as the researcher performing a narrative analysis of themselves in relation to a noteworthy phenomenon.

Two narratives aim to capture the culture of my experience in being witness to the death of my child. They contain a raw account of my emotions and senses at the time of the event and beyond. Pace (2012) identified that the narrative explains how and why the phenomenon occurs and is thus part of the method of my analysis. These narratives are sometimes graphic in nature to put across the reality of my child's death, and my subsequent grief.

This learning process involves interaction between the environment, the learner, and the instructor. In this instance the instructor is the event that occurred. However, learning is forgotten without reflection, where concepts arise from feelings and thoughts (Gibbs, 2013). Moreover, it is for the reader to determine the authenticity of the narrated experience based on their own interpretation (Ellis et al., 2011). From the narrative, one can attempt to evoke a response from the reader; do you as the reader feel a vicarious emotion? Comparison between the story and the reader's life experience gives theoretical validation (Ellis, 2004).

The Toi Ohomai Institute of Technology Human Ethics Committee provided advice and guidance regarding ethical concerns relating to other family members. Not only is consent to undertake this study obtained from every member of my close family, pseudonyms also protect their identity and that of the deceased. Ellis et al. (2011) observed that researchers do not live in isolation. The author may implicate others by undertaking their research, so it is important to protect others and seek their consent to the study.

The study takes me back to that night. Thus, self-care breaks were an essential part of my study when revisiting the horror of the event. This also involves seeking psychosocial support when required, taking account of any re-traumatisation. There is a note of caution to readers who may have previous experience of child bereavement as the narratives may evoke distress or discomfort.

\section{Data analysis}

The following two narratives describe a complex weave of past and present, whilst the research can offer advice for future emergency response when working alongside bereaved parents.

\section{The event}

\section{Grief support from the family social network system}

The narratives are analysed using two perspectives; one being personal narratives as data to reflect upon my personal senses at the time of the traumatic event. Secondly, analysis of the data considers emerging themes from the narratives. I thus interpret my personal experiences through recall whilst considering a professional response from emergency personnel. Recall is a complex cognitive process of past, present, and future. Gallagher and Zahavi (2014) described analysis of any experience (perception, memory, imagination) containing a reference to past moments of experience, a primal impression of what is happening and an anticipation of what may happen. 
The first level of analysis is an iterative process, where additional life events have occurred. Such events can alter a person's perspective of what occurred, as identified by Tolich and Davidson (2011). The second level of analysis is an objective thematic analysis taken from the narrative; this creates another view of the phenomenon. The researcher takes a step back from their narrative when using analytical autoethnography. Themes from the text (either within or across the text) are ideas that offer a deeper explanation of the content. Parts of the narrative may highlight an emerging issue (Ellis, 2004).

Coded themes about emergency personnel present what interactions took place, thereby identifying emerging messages. The analysis inspects the personal impact of professional interaction on myself at a time of trauma. I can then consider training and education for professional responders when managing crisis intervention.

Emerging themes from the narrative text can plan a theory (Pace, 2012). This theory explains how and why a phenomenon occurred and is not a test for generalisability. The phenomenon observed in this instance, is my experience of traumatic parental bereavement.

\section{Findings}

I have chosen parts of the narrative that describe the phenomena and once again remind readers of the evocative content and the impact it may have on them as the reader. The first piece of chosen text from the narrative expresses my feelings whilst in a state of anxious panic awaiting the arrival of first responders:

With time of the essence, feelings of panic were rising inside, being reminded constantly by the emergency controller to keep calm.

As the parent of a child whose life was ebbing away before my eyes, I was reliant on emergency response personnel to assist in the resuscitation of my child. With no signs of life from them, I started to panic from rising anxiety as time was quickly passing by without professional first responder input.

Living in rural Aotearoa New Zealand brings complications when emergency services are not easily accessible, as opposed to living in the city. Through research, I discovered that it is rare for a child who is not breathing and whose heart has stopped, to survive. Not all cardiac arrests are recoverable, especially in children. Jewkes (2001) explained that most cardiac arrests in young children are secondary to hypoxia, (inadequate oxygenation of the blood), or shock. Brindis et al. (2011) stated, in their research, that out of almost four hundred child patients needing resuscitation, only four $(1.2 \%)$ responded. Paramedics often find the electrical heart rhythm to be missing in young children who are not breathing, making it impossible to revive them. Upon reflection, this has changed my assumption that first responders can succeed in reviving a young child in a state of hypoxia. This is not a message I would have wanted to know but it creates an understanding whilst managing personal parental grief.

For months after the event, I agonised over my actions that night, wondering if I could have done things differently:

After the event, I discovered it had taken seventeen minutes for the first ambulance to arrive at the location. The volunteer emergency responder jumped from their vehicle asking the way into the home and raced off from the dark towards the light of our downstairs rumpus room area where the ranch slider entrance was ajar. Following behind the first emergency responder, phone still in hand, we reached the bedroom. On seeing $X^{\prime}$ 's collapsed condition, the volunteer responder asked me to return to the vehicle to search for a defibrillator machine in the rear left-hand side of the ambulance. 
Months following the death of my child, I sent a letter of complaint to the local emergency ambulance service expressing concern over the time delay before the arrival of the emergency ambulance responders. This included the initial responder failing to take a defibrillator into the house, as the emergency controller informed them that my child had no signs of life. Although this would not change the outcome for my child, it was comforting that GPS navigation systems are now fitted in all ambulance vehicles and training undertaken in relation to the defibrillator incident.

But this was not the only concern relating to the emergency response:

I cannot remember any further questions asked at that point, into the night's events, but can remember a diminutive guy appear from nowhere to say he was sorry and would leave a brochure on the sideboard. As a traumatised parent it was difficult to give him attention at the time.

Aotearoa New Zealand has a victim support system. At the time of a sudden death, an amateur volunteer will attend a traumatic event to offer support. Unfortunately, the victim support volunteer failed to communicate important information that might prepare a bereaved parent for negotiation with a coroner over the issue of performing an autopsy. The coronial service in Aotearoa New Zealand sets out the right for family to challenge the need for an autopsy within 48 hours of an unexplained death. This right was not explained to a confused and disoriented bereaved parent. Although not affecting my family, I am aware this mistake may have a large impact on others whose cultural needs are different. For example, Hudson et al. (2008) explored the impact of Māori culture on forensic science practice. To be away from, and not have access to, their loved one's body may cause further distress.

I refer earlier, to the poor outcome for the survival of most young children who have hypoxia. I have reflected on this poor survival rate and recognise it is not always possible for first responders to save the life of a child who is not breathing. Thus, control over the life or death of a child with hypoxia can be random. So, what are first responders able to control at the scene of a hypoxic child? There is the important attempt to save the life of a young child, but it is also important to care for traumatised parents.

The impact of the trauma is described in this next passage:

Someone distracted me from my child's room, although, really wanting to remain there with them. A family member had arrived at the house. I remember standing on steps in the hallway before walking down to greet them. People were around whilst they asked what had happened; trying to tell them but failing to, I saw the distressed, yet inquisitive look on their faces of disbelief. Sounding distressed they questioned, "Why aren't you crying?" At the time there were no words due to my disorientation.

A sudden death is often traumatic where people may be unable to process the shock immediately and their body may react in a series of symptomatic responses. Dyregrov and Dyregrov (2008) described bereaved parents' reaction to their child's death as a sense of unreal, being in a dream-like state, and wishing it were not true. There may also be a sense of remorse and blaming of themselves about not doing more to prevent the death or of the ways they reacted at the time of death.

Dyregrov and Dyregrov (2008) described the death permeating the individual when it unfolds as a trauma, where the memory of the event is etched into their mind due to the intensity of the shock and emotion. When the shock is so intense, the mind seeks to protect and does not absorb the information in one go. Often reactions to shock take over, such as shaking, palpitations, dizziness, 
numbness, chills, and nausea; people often become disoriented. This may continue for some time beyond the initial event with many chaotic racing thoughts, and the memory of bereaved parents affected where they are unable to recall the events.

It is so important that others can take charge during a period of temporary disorientation for a parent whose ability to function well is affected by a crisis. The emergency controller took charge in obtaining information from me by asking me to be calm, enabling me to contribute to the efficiency of the response.

\section{Professionals taking control}

The ability of other professionals to take control was later observed by myself and commented on. Following the arrival of the ambulance, a uniformed police officer took control of the immediate scene, information, and guided my partner and I to avoid hampering the emergency response. This enabled paramedics to take control of the resuscitation effort. Police detectives investigated an unexplained death following the initial crisis for a coronial inquiry and any criminal proceedings, but did so whilst maintaining our dignity.

It is important to note that a person in a traumatic crisis may be disorientated and reliant on the skills of professional first responders performing their roles, including communicating what is happening with their loved one. I reflect upon this approach with gratitude; professionals need to guide and direct traumatised parents. Such parents are in no fit state to organise necessary services to manage the next stage of the event, the removal of the young child's body for an autopsy. Saari (2005) describes the importance of a trained crisis intervention response allowing bereaved parents time to respond to the event in their own way.

Professional intervention is required to establish the circumstances surrounding a death, yet this is performed during a time when traumatised parents are at their most vulnerable. It can offer comfort, though, when parents who are not responsible for their child's death are questioned by police and are determined as innocent of any crime. Heltne et al. (2016) described the routine examination of the death scene following a sudden infant death in Norway. They explained that the death scene investigation determines the cause and gathers new knowledge of the death scenario. The death scene investigation also performs an important role in attributing responsibility and confirmation of the cause of death. This may reduce rumination for some bereaved parents in believing that they may have been in some ways responsible for the death, even if they are not.

\section{The need for a sensitive approach}

Having described the benefit of first responders taking control for traumatised parents, it is still important for professionals to manage their care sensitively. Janzen et al. (2004) stated that bereaved parents still need some sense of control. The police enabled me to carry my child's body to the undertaker's hearse and allowed the family to remain near my child at the mortuary, although not in the same room. The police obliged as much as they were able to, given their protocols to protect evidence of what had occurred that night. Regardless of protocols, the police detectives allowed controlled access to my child's body before the temporary removal to an autopsy. Janzen et al. (2004) noted this as an opportunity for the bereaved parents to begin grieving.

The efficiency of determining an interim assessment of the likely cause of death was another important factor in supporting the welfare of my family and me. Less than twenty-four hours after the event, the police detectives returned to the family to inform of the initial outcome of their investigation. They explained their belief that my child's death was due to misadventure, ruling out parental involvement in the cause of death. Their assessment of the event was welcomed with relief as there was vindication from the 
suspicion of a young child's death at our hands and grateful that the professionals had demonstrated respect for the family, and my child's body. On reflection I felt an empathic response by professional responders and, in turn, the family developed trust and confidence in their actions.

\section{Important messages for professional services working alongside traumatised and bereaved parents}

The sudden death of a child is observed by Dyregrov and Dyregrov (2008) and Feigelman et al. (2012) as a devastating event for a parent. Its impact may continue long after the initial event, especially when such grief becomes complicated due to factors related to the event, the degree of trauma and the responses of others. It is thus important that emergency response personnel have the necessary attending skills described by Aho et al. (2010) as affect, aid, and affirmation. Hermansson and Webb (2009) described one of the skills in counselling as attending. Some strengths involved with this skill are respect, dignity, an open attitude, and an ability to actively listen. This means putting one's thoughts aside in attending to the needs of the individual.

Aho et al. (2010) explained the meanings of affect, aid and affirmation as skills. 'Affect' is the professional's presence in which bereaved parents feel listened to and understood, creating an active listening environment (Hermansson \& Webb, 2009). 'Aid' involves guiding parents through tasks that must occur following a sudden death. Such guidance is essential due to the potential for confusion and disorientation of the parents. Combined, 'affect' and 'aid' should ensure that the parents or their representatives have understood the procedures required postevent. 'Affirmation' enables parents to start processing the psychological reality of their child's death. Fulbrook (2015) explained how bereaved parents must begin to adapt to the sudden change of shattered assumptions. This communicative role plays a significant part in how the impact of the death may affect the parent's future wellbeing. This approach is emphasised by Saari (2005) in advocating for professional training and response toward bereaved parents. Active listening and rejecting one's own impulses to rescue the parents are essential. Stating words such as "it will be alright," "things will improve," or "they have gone to a better place," are demeaning for parents. Such comments may make the responder more at ease but may leave questions in the minds of parents, reinforcing their pain and even a sense of guilt that they should have parented better.

The initial response to parental trauma is an important start to a long journey, it is important for emergency responders to display empathy and effective control to enable the experience of grief to occur without further trauma. It is important for all emergency responders and professional supporters to be competent in advanced communication skills to manage the reactions and needs of bereaved parents. Trained emergency responders can take control of a traumatic event when others are unable to; this approach offered support to me and my partner, and a sense of protection and security.

\section{Implications}

Emergency personnel attending a traumatic death scene must consider the needs of those who are witness to the event, such as the long-term welfare of parents involved in the resuscitation effort of their child. This approach plays an important role in the later grief and healing of those affected. The key messages from my research are:

- Bereaved parents can be in such a state of shock from the trauma that they are no longer in control of their emotions, senses, and cognition.

- There is a valuable role for professionals to take control of the situation when parents are in shock and lacking 
cognitive ability. They can actively listen to parents. Their guidance is important for the legal processes, and for the long journey of grief bereaved parents will have to walk.

Effective communication can reassure the family and start to build family trust in the legal procedures occurring, as such practice is part of the ongoing journey for the family post-bereavement. This can offer an understanding of bereaved parents' rights during clinical processes such as an autopsy. Aid in guiding the family through necessary procedures reinforces their experience of feeling supported during a period of confusion and disorientation. Offering respect and empathy can enable the start of the grieving process due to bereaved parents experiencing support in such traumatic circumstances.

I have reflected on empirical and theoretical research and drawn on personal learning to offer advice to the community of professional emergency first responders. Being a dualinsider researcher has offered further insight into the world of bereaved parents that is often recorded as a third person researcher. This research is an account of the experience of loss from an individual who works within the field of social work and first response. This approach is rarely used, yet can offer greater insight to research knowledge.

Accepted 20 July 2021

Published 1 September 2021

\section{References}

Aho, A., Tarkka, M., Astedt-Kurki, P., \& Kaunonen, M. (2010). Health care personnel's experiences of a bereavement follow-up intervention for grieving parents. Scandinavian Journal of Caring Sciences, 25(2), 373-382. http:// dx.doi.org/10.1111/j.1471-6712.2010.00837.x

Brindis, S., Gausche-Hill, M., Young, K., \& Putnam, B. (2011). Universally poor outcomes of paediatric traumatic arrest: A prospective case series and review of the literature. Paediatric Emergency Care, 27(7), 616621. http://dx.doi.org/10.1097/PEC.0b013e31822255c9

Dyregrov, K., \& Dyregrov, A. (2008). Effective grief and bereavement support. Jessica Kingsley Publishers.

Ellis, C. (2004). The ethnographic I. Altamira Press.
Ellis, C., Adams, T., \& Bochner, A. (2011). Autoethnography: An overview. Forum: Qualitative Social Research, Sozialforschung. http://www.qualitative-research.net/ index.php/fqs/article/view/1589/3095

Feigelman, W., Jordan, J., Mclntosh, J., \& Feigelman, B. (2012). Devastating losses. Springer Publishers.

Fulbrook, T. (2015). Bereavement and parents who have experienced the sudden death of a child [Unpublished doctoral dissertation]. Walden University.

Gallagher, S., \& Zahavi, D. (2014). Phenomenological approaches to self-consciousness. The Stanford encyclopaedia of philosophy. https://plato.stanford. edu/archives/win2016/entries/self-consciousnessphenomenological/

Gibbs, G. (2013). Learning by doing: A guide to teaching and learning methods (1st ed.). Oxford Brookes University. https://thoughtsmostlyaboutlearning.files.wordpress. com/2015/12/learning-by-doing-graham-gibbs.pdf

Heltne, U., Dyregrov, A., \& Dyregrov, K. (2016). Death scene investigation: Parents' experiences. Scandinavian Journal of Forensic Science, 22(2). http://dx.doi. org/10.1515/sjfs-2016-0009.

Hermansson, G., \& Webb, S. (2009). Eclectic counselling: Working with an integrated model (3rd ed.). Inside-Out Books.

Hudson, M., Allan, C., Bedford, K., Buckleton, J., \& Stuart, K. (2008). The impact of Māori cultural values on forensic science practice in New Zealand. Journal of Forensic Sciences, 53(2), 380-383. http://dx.doi.org/10.1111/ j.1556-4029.2008.00661.x

Janzen, L., Cadell, S., \& Westhues, A. (2004). From death notification through to the funeral: Bereaved parents' experiences and their advice to professionals. OMEGA - Journal of Death and Dying, 48(2), 149-164. http:// dx.doi.org/10.2190/6xuw-4pyd-h88q-rq08

Jewkes, F. (2001). Current topic: Prehospital emergency care for children. Archives of Disease in Childhood, 84(2), 103-105.http://dx.doi.org/10.1136/adc.84.2.103

Kim, J. (2016). Understanding narrative inquiry: The crafting and analysis of stories as research. Sage.

Mendez, M. (2013). Autoethnography as a research method: Advantages, limitations, and criticisms. Colombia Applied Linguist Journal, 15(2), 279-287. http://www. scielo.org.co/pdf/calj/v15n2/v15n2a10.pdf

O'Hara, M., Carter, C., Dewis, P., Kay, J., \& Wainwright, J. (2014). Successful dissertations. Bloomsbury.

Pace, S. (2012). Writing the self into research: Using grounded theory analytic strategies in autoethnography. Journal of Writing and Writing Courses, 13, 1-15. http:// www.textjournal.com.au/speciss/issue13/Pace.pdf

Regehr, C. (2011). Crisis theory and social work treatment. In F. J. Turner (Ed.), Social work treatment: Interlocking theoretical approaches (pp. 134-143). Oxford University Press.

Saari, S. (2005). A bolt from the blue: Coping with disasters and acute traumas. Jessica Kingsley Publishers.

Tolich, M., \& Davidson, C. (2011). Getting started: An introduction to research methods. Pearson. 